\title{
Modernização, envelhecimento e infoexclusão em Portugal
}

Modernization, aging and info-exclusion in Portugal

Modernización, envejecimiento e info-exclusión en

Portugal

Alexandre Morais Nunes

RESUMO: O envelhecimento é um desafio que, nos últimos anos, tem sido objeto de estudo na sociedade moderna. Todos tentam perceber e compreender o envelhecimento e intervir para reduzir seus efeitos naturais, evitando a exclusão da pessoa idosa; pelo contrário, promovendo a sua integração na sociedade. A questão que se coloca em Portugal e nas sociedades mais modernas é o grande desenvolvimento das tecnologias de informação. Estes processos geraram, em Portugal, uma maior dependência para tratar de assuntos quotidianos, como o acesso aos serviços bancários, finanças, marcação de consultas, levantamento de medicamento nas farmácias e até compras. No presente artigo, destaca-se a questão da infoexclusão e da infointervenção junto do idoso, e apresentam-se alguns resultados do uso de tecnologias de informação por parte dos idosos em Portugal.

Palavras-chave: Envelhecimento; Política social; Pessoa idosa; Tecnologias de Informação; Infoexclusão. 
ABSTRACT: Aging is a challenge that in recent years has been the object of study in modern society. We all try to understand and understand aging and intervene to reduce its natural effects by avoiding the exclusion of the elderly and, instead, by promoting their integration into society. One issue that arises in Portugal and in more modern societies is the great development of information technologies. These processes led to greater dependence in Portugal on everyday matters such as access to banking services, finances, appointment of appointments, collection of medicine in pharmacies and even purchases. In this article, we highlight the issue of infoexclusion and infointervention among the elderly and present some results of the use of information technologies by the elderly in Portugal.

Keywords: Aging; Social policy; Elderly people; Information Technologies; Infoexclusion.

RESUMEN: El envejecimiento es un desafío que en los últimos años ha sido objeto de estudio en la sociedad moderna. Todos intentamos percibir y comprender el envejecimiento e intervenir para reducir sus efectos naturales evitando la exclusión de la persona mayor y por el contrario promoviendo su integración en la sociedad. Una cuestión que se plantea en Portugal y en las sociedades más modernas es el gran desarrollo de las tecnologías de la información. Estos procesos generaron en Portugal una mayor dependencia para tratar asuntos cotidianos como el acceso a los servicios bancarios, finanzas, marcación de consultas, levantamiento de medicamentos en las farmacias e incluso las compras. En el presente artículo se destaca la cuestión de la info-exclusión y de la información sobre el anciano y se presentan algunos resultados de la utilización de tecnologías de información por parte de los ancianos en Portugal.

Palabras clave: Envejecimiento; Política social; Personas mayores; Tecnologías de Información; Infoexclusión.

\section{Introdução}

O envelhecimento é uma realidade, em países modernos e mais desenvolvidos, que traz algumas limitações físicas e mentais próprias da idade avançada. 
Por essa razão, o envelhecimento é visto como um problema da sociedade que limita a atividade das pessoas. Nesse sentido, em 1989, Walsh definiu o modo como é sentida a chegada dos efeitos do envelhecimento por parte dos cidadãos, referindo que "a velhice é temida quase tanto como se teme não viver suficiente para a alcançar" (Walsh, 1999, p. 312).

As políticas e estratégias definidas para promover um envelhecimento ativo, saudável e integrado da pessoa idosa na sociedade é uma prioridade para o Governo de Portugal. Como refere Nunes (2017, p. 152):

Em Portugal, no ano passado, promoveu-se um investimento na promoção da saúde dos idosos e na capacidade de resposta do Serviço Nacional de Saúde às suas necessidades na doença aguda, crônica, na reabilitação e na reintegração social, o que é visto pelo Governo como um investimento em saúde que mantém uma população idosa monitorizada e ativa que vive mais e melhor, e gera menos despesa pública. A partir destas estratégias, o Governo pretende criar as condições necessárias para promover uma política de futuro para o modo de envelhecer que passa por uma aposta real nas necessidades e na correção dos problemas atuais e prevenção dos futuros.

O desenvolvimento das tecnologias e do mundo da informação digital é uma constante nas sociedades modernas (Tredinnick, 2008). O acesso aos serviços públicos e à informação (saúde, finanças, segurança social), em Portugal, foi simplificado com uma grande facilidade de acesso através de plataformas eletrônicas acedidas através de um computador ligado à internet. $\mathrm{O}$ problema que se coloca é que muitos dos idosos não têm a capacidade de acessar todos esses meios, o que pode gerar o risco de ser mais um modo que pode desqualificar os idosos na sociedade, conhecido como infoexclusão.

Este artigo apresenta um estudo descritivo-exploratório da realidade portuguesa, com o objetivo de percepcionar e alertar os factos relacionados com a adoção dos idosos às tecnologias de informação, tendo por base o fenômeno da infoexclusão. 


\section{O Envelhecimento populacional em Portugal}

Em Portugal, o envelhecimento da população é uma realidade inegável que tem crescido exponencialmente nas últimas décadas, como consequência do aumento da longevidade (Nunes, \& Nunes, 2016), associado a um crescimento da esperança média de vida que cresceu mais de 30 anos (Ferrinho, Bugalho, \& Pereira, 2001).

O envelhecimento da população é uma realidade transversal a todos os países desenvolvidos, que foi resultado da melhoria das condições de vida, do desenvolvimento da tecnologia e do conhecimento científico, bem como da adoção de práticas de saúde saudáveis, e do estímulo à prática da atividade física (esporte) (Nunes, 2017). Além desses fatores, de acordo com Quaresma e Ribeirinho (2016), a maior longevidade está associada simultaneamente a uma melhor educação, melhor acesso ao emprego, à proteção social (na doença e na insuficiência econômica), ao aumento dos rendimentos e à urbanização dos territórios.

Apesar do desenvolvimento de uma cultura urbana em torno do envelhecimento e do fortalecimento das relações sociais exigidas, não deixam de estar associados alguns riscos às pessoas mais idosas (Bruno, 2006).

O envelhecimento populacional não diz respeito apenas ao aumento do número de pessoas com mais idade, mas também está relacionado com a ocorrência de outros fenômenos demográficos que poderão ter consequências futuras na sociedade (Brito, 1993).

Tabela 1- População jovem e idosa em 1960 e em 2001

\begin{tabular}{c|c|c|c|c|c}
\hline \multirow{2}{*}{} & População & \multicolumn{4}{|c}{ Grupos etários } \\
\cline { 2 - 6 } & Total & $0-14$ anos & $\%$ & $>65$ anos & $\%$ \\
\hline $\mathbf{1 9 6 0}$ & 8.889 .392 & 2.591 .955 & $29,2 \%$ & 708.569 & $8,0 \%$ \\
\hline $\mathbf{1 9 7 0}$ & 8.611 .125 & 2.451 .850 & $28,5 \%$ & 832.760 & $9,7 \%$ \\
\hline $\mathbf{1 9 8 1}$ & 9.833 .014 & 2.508 .673 & $25,5 \%$ & 1.125 .458 & $11,4 \%$ \\
\hline $\mathbf{1 9 9 1}$ & 9.867 .147 & 1.972 .403 & $20,0 \%$ & 1.342 .744 & $13,6 \%$ \\
\hline $\mathbf{2 0 0 1}$ & 10.356 .117 & 1.656 .602 & $16,0 \%$ & 1.693 .493 & $16,4 \%$ \\
\hline $\mathbf{2 0 1 1}$ & 10.562 .178 & 1.572 .329 & $14,9 \%$ & 2.010 .064 & $19,0 \%$ \\
\hline
\end{tabular}

Fonte: CENSUS (2011) 
Em Portugal, no ano de 1960, a proporção da população idosa (com mais de 65 anos), representava cerca de $8 \%$ da população total e em 2001 esse valor duplicou atingindo 19\% em 2011. Relativamente à população jovem (idade compreendida entre os 0 e os 14 anos), no ano de 1960 representava 29,2\% do total da população e em 2011 apenas 14,9\%. A figura seguinte apresenta a tendência observada.

Figura 1 - Tendência evolutiva da população jovem e idosa em Portugal

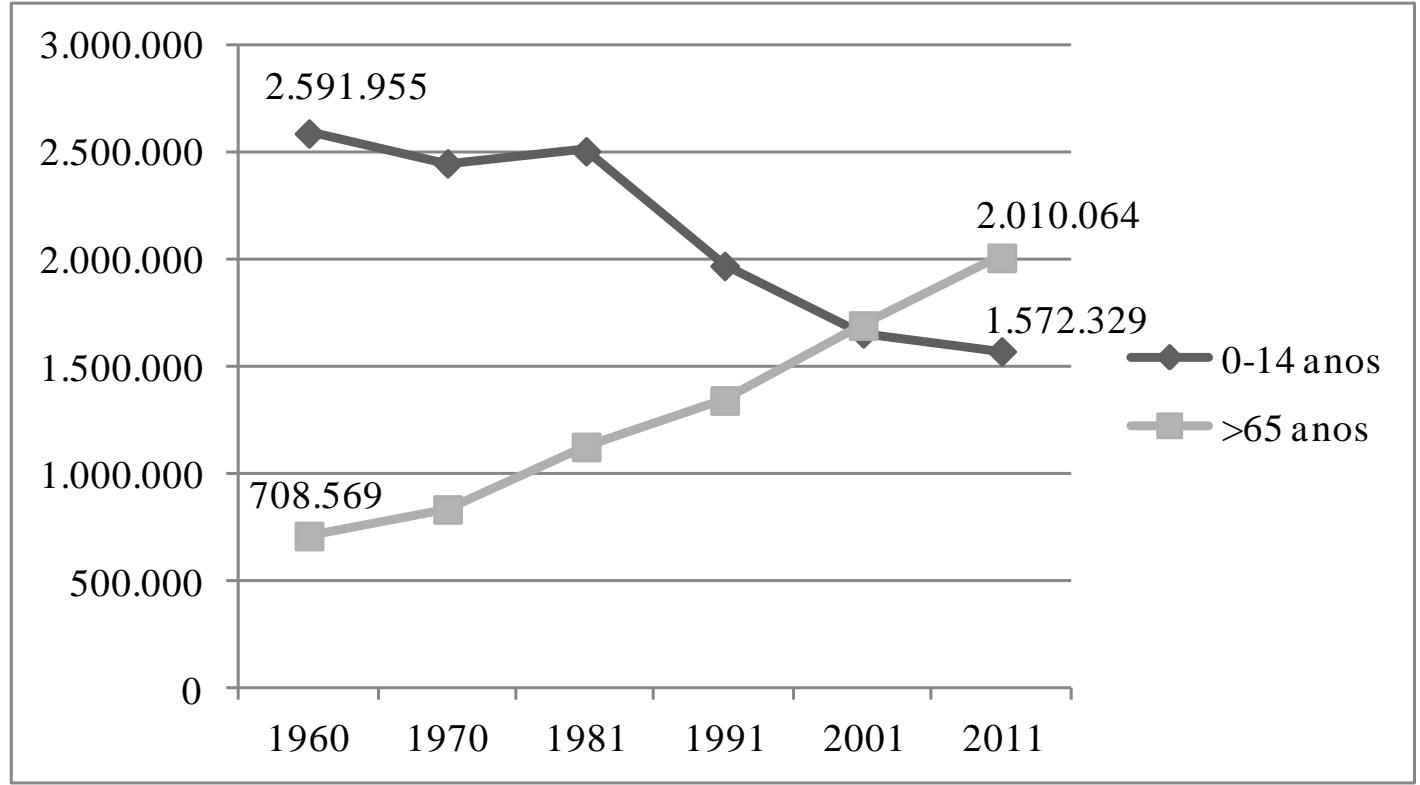

Fonte: INE (2015a)

Analisando entre cada grupo etário, verifica-se que a população jovem sofreu uma redução de $39,3 \%$ enquanto que a população idosa registrou um crescimento de $183 \%$.

Os dados preliminares relativos ao ano 2015 não são encorajadores e apontam que mais de $20 \%$ da população portuguesa é idosa (INE, 2015a). As alterações demográficas registradas no presente poderão ter consequências agravadas no futuro pelas baixas taxas de fecundidade e natalidade, e pela elevada emigração da população portuguesa em idade ativa (Nunes, 2017; Quaresma, \& Ribeirinho, 2016).

Em Portugal, existem ainda outros problemas que são a assimetria populacional entre o Litoral e o Interior, este tendo uma população mais envelhecida e com menos recursos financeiros (Nunes, 2017) e ainda existem assimetrias nas condições de vida das pessoas que integram o grupo etário de mais de 50 anos. Em respeito a esta última questão, Quaresma e Ribeiro (2016, p. 31) realizaram um estudo e concluíram que: 
A análise das condições de vida e das expectativas das gerações que constituem o grupo das pessoas de 50 e + anos é reveladora da importância das transformações sociais, económicas e políticas, no aumento da longevidade, e na qualidade do envelhecer.

Ainda em relação às questões demográficas, Portugal tem vindo a receber vários alertas sobre os riscos demográficos para a sociedade associados ao envelhecimento e à fraca natalidade registrada:

- O Instituto Nacional de Estatística tem alertado que as alterações demográficas são claras e antevê projeções arrepiantes para o futuro com base nos dados de 2015 que apontam cerca de $20 \%$ de idosos na população;

- A Organização das Nações Unidas (ONU) prevê que, em Portugal, no ano 2050, apenas $20 \%$ da população seja constituída por crianças e jovens com idade compreendida entre os 0 e os 14 anos, recordando que esta proporção em 1960 era de 37\%, e no ano 2000, de 30\%;

- O Eurostat (Departamento de Estatística da União Europeia) também definiu um cenário assustador e destaca que, em 2050, a percentagem de idosos em Portugal será superior a 32\% (quarto país da Europa com maior percentagem de pessoas idosas).

\section{O fenômeno do Envelhecimento: estereótipos, perspectivas e realidade}

Hoje, com o aumento da longevidade da população nas sociedades modernas, torna-se muito difícil estabelecer um marco para estipular uma idade que caracterize o início da velhice. Em termos estatísticos, é tida em conta a idade de 65 anos como uma referência (idade considerada na aposentação em Portugal, por exemplo) (Duarte, et al., 2005).

Muitas vezes, o envelhecimento da população é entendido como sendo um paradoxo (Novais, 2008) e é analisado em dois pontos de vista: um positivo e outro negativo. Esta métrica é referida em vários posicionamentos teóricos, em que se destaca a Organização das Nações Unidas (ONU, 2002) e os trabalhos de Walsh (1989), Nazareth, (1998), Sequeira (2007). 
De um ponto de vista negativo, a sociedade encontra no envelhecimento apenas um conjunto de problemas que reduzem a atividade da pessoa e que as desqualifica individual e socialmente. Walsh (1989) refere esta questão, ao afirmar que:

A sociedade ocidental estereotipa e desqualifica os idosos, considerando-os senis, retrógrados, rígidos, inúteis, chatos e aborrecidos.

Ainda em um ponto de vista negativo, o envelhecimento é também visto na sociedade moderna como um estado da vida caracterizado por isolamento, solidão, pobreza, doença, demência física e social, e incapacidade de gerir a sua própria vida e de gerar valor para a sociedade (Sequeira, 2007). Os investigadores ingleses determinaram uma expressão para caracterizar esta fase conhecida como "Disengagement teory", e que tem um significado de "desligamento da sociedade".

A teoria do "Disengagement" afirma que o envelhecimento é uma retirada mútua inevitável ou desengate, resultando de uma diminuição da interação da pessoa idosa com o meio social a que pertence (Cumming, \& Henry, 1961). Esta perspectiva considera natural e aceitável que as pessoas mais velhas se retirem da sociedade (Ebersole, 2005), e justifica esse desligamento com 9 postulados (Cumming, \& Henry, 1961), que se apresentam de forma resumida na tabela seguinte:

Tabela 2 - Postulados da perspetiva negativa de envelhecimento

Postulado 1: todos os idosos esperam a morte, e nesse processo as suas habilidades vão sendo perdidas. Como resultado, cada pessoa vai perdendo laços com a sociedade.

Postulado 2: Os idosos têm menor interação com a sociedade e, por conseguinte, o desengate torna-se a um processo de autoperpetuação.

Postulado 3: Os homens têm um papel mais ativo na sociedade e, por isso, o desengajamento difere entre homens e mulheres.

Postulado 4: a vida do indivíduo é pontuada por mudanças no seu ego. $\mathrm{O}$ envelhecimento produz uma mudança no ego, no conhecimento e promove a perda de habilidades que leva a pessoa a deteriorar-se.

Postulado 5: a retirada deve ocorrer quando o indivíduo e a sociedade estão prontos.

Postular 6: O principal papel do homem é o trabalho e o da mulher é o casamento e a família. Quando os indivíduos abandonam estes seus papéis principais, perdem drasticamente espaço na vida social e, por isso, em crise interior sofrem uma desmoralização. 
Postulado 7: a prontidão para a retirada ocorre quando um indivíduo está ciente da falta de vida e da escassez de tempo.

Postular 8: o desprendimento é iniciado pela redução das interações.

Postulado 9: o desprendimento é independente da cultura.

Fonte: Cumming, \& Henry (1961)

Essa teoria formulada por Cumming e Henry, em 1961, não tem qualquer cabimento na sociedade atual. Desde a sua criação e até hoje tem sido alvo de fortes críticas que focam a sua perspectiva inata, universal e unidirecional (Bengtson, et al., 2009), tendo crescido, por sua vez, a perspectiva positiva do envelhecimento.

A teoria do desengajamento é uma das três principais teorias psicossociais. As outras duas principais teorias psicossociais são a teoria da atividade e da teoria da continuidade (Bengtson, et al., 2009).

As perspectivas positivistas defendem que o envelhecimento não é uma doença, nem é propriamente o fim de um ciclo, mas, pelo contrário, uma realidade atual que tem crescido com o aumento das pessoas idosas e que deve ser encarada como uma fase da vida (Fernandes, 1999; Freire, 2000; Nunes, 2017). A este respeito, Nazareth (1998, p. 135) complementa:

O envelhecimento não se trata de uma nova praga ou doença, mas de uma simples constatação quantitativa: o número de pessoas idosas estará a aumentar preocupantemente.

De acordo com as teorias positivistas, o envelhecimento é uma etapa da vida humana, caracterizado por uma deterioração irreversível das capacidades e funcionalidades vitais que se reconhecem num adulto, que se manifestam por um declínio visível e vulnerabilidade acentuada. Isso não impede que seja bem-sucedido e integrado de forma positiva com a sociedade (Albert, et al., 1995; Sousa, \& Figueiredo, 2003). Do ponto de vista positivo, os idosos contribuem de uma forma muito valiosa para a vida social e econômica da sociedade, em interação com as suas famílias, desenvolvendo atividades, fazendo voluntariado, e apoiando seus filhos (Lima, 2010; Silva, 2006). Segundo Fernandes (1999), o processo de envelhecimento bem-sucedido contempla dimensões como: a emocional, que trata da capacidade para lidar com as limitações físicas e pessoais; e a cognitiva/comportamental, que reflete a capacidade para processar os seus problemas de forma consciente. 
Esta perspectiva positiva vai ao encontro do que conhecemos como envelhecimento ativo. Existem múltiplas definições para este conceito, sendo a mais clara e internacionalmente aceite, a definida pela Organização Mundial de Saúde (OMS, 2012, p. 12), que classifica o envelhecimento ativo como um processo de oportunidade para a participação e segurança que promove a saúde e o bem-estar das pessoas que envelhecem, garantindo os seus direitos e a sua integração social ao longo da vida, participando de forma ativa na vida social, econômica, cultural, espiritual e cívica.

O envelhecimento saudável e ativo é um desafio atual e futuro para as sociedades desenvolvidas, que tem efeitos diretos na economia de um país, e que determina a forma como decorre a participação individual e coletiva dos cidadãos idosos, envolvendo diversos sectores (Fonseca, 2005).

A intervenção social para promover o envelhecimento deve ser de âmbito estrutural e envolver todas as classes sociais, órgãos de governo e população e requer uma reforma que não passe apenas pela satisfação das necessidades humanas afetadas pelo envelhecimento, mas, sim, por uma estratégia de preventiva com integração da pessoa idosa nas atividades de vida diária, acompanhamento médico de proximidade na atenção primária e não na urgência de um hospital e uma resposta alargada às necessidades físicas e psicológicas, fazendo com que o idoso se sinta útil e integrado (Carvalho, 2012). É apenas com estes tipos de respostas que se pode potenciar a participação ativa do idoso na sociedade. Ratificam essas ideias, o seguinte fragmento de Quaresma e Ribeirinho, (2016, p. 38):

As políticas de envelhecimento (...) constituem uma resposta, urgente e necessária, cuja eficácia depende da devida articulação com as diferentes políticas sociais sectoriais. O conhecimento, a experiência e a reflexão no contexto das sociedades mais envelhecidas deu lugar à construção do conceito de Envelhecimento Ativo, postulado pela Organização Mundial da Saúde (OMS) e também pela Organização para a Cooperação e Desenvolvimento Económico (OCDE), ainda que, com algumas diferenças, acolhendo a interdependência entre envelhecimento/desenvolvimento humano/ desenvolvimento social/políticas sociais. 
As intervenções orientadas para a participação do idoso na sociedade dizem respeito à ação de política social desenvolvida pelo Governo, respectiva vontade da sociedade e cabimento orçamental para prover os recursos necessários (Carvalho, 2013). Importa aqui ressaltar o papel que o Governo de Portugal tem realizado com o desenvolvimento e promoção de uma estratégia para o desenvolvimento global e ativo, adaptados às necessidades da sociedade e dos próprios idosos, entre as quais se destacam, de acordo com Nunes (2017, pp. 149-150):

A Integração das pessoas desempregadas nas comunidades locais, desenvolvendo atividades e programas em regime de voluntariado junto das pessoas mais idosas, com o objetivo de torná-las mais ativas, mais seguras, partilhar experiências, e promover estilos de vida saudáveis;

A Inserção social dos idosos em programas de serviço comunitário junto da população mais desfavorecida;

O Lançamento de programas de apoio ao cidadão idoso, nomeadamente aqueles que têm problemas de mobilidade e que necessitam de um apoio especial nas suas práticas do dia a dia;

A Promoção do financiamento da construção de centros de saúde e unidades de apoio ao idoso (centros-dia);

O Desenvolvimento de atividades para integração da população;

A Eliminação das barreiras arquitetônicas que condicionam a mobilidade nos espaços públicos;

A colaboração com as equipas de saúde, disponibilizando transporte para o acompanhamento no domicílio.

A concepção dessas medidas teve por base uma pesquisa realizada em 2016 pelo Governo de Portugal (nomeadamente pela constituição de um Grupo de Trabalho repartido por elementos do Ministério da Saúde e do Ministério do Trabalho e da Segurança Social). Estudos anteriores revelavam uma escassez de conteúdo e incidiam apenas nos impactos do envelhecimento nas contas do Estado, no emprego e não tinham em consideração as relações sociais no envelhecimento, a autonomia dos cidadãos, a perspectiva da família, a partilha de informação, o convívio, as atividades organizacionais. A este respeito, Quaresma (2008, p. 27), refere mesmo que não foi tido em conta nos estudos realizados em Portugal: 
(...) a lógica de construção/consolidação de laços de identidade, favorecendo as relações interpessoais significantes e estruturantes da própria identidade dos lugares habitados (Quaresma, 2008, p. 27).

O envelhecimento orientado numa direção positiva pode ser uma oportunidade para viver de uma forma saudável e autónoma, com participação ativa na sociedade. A este respeito, Nunes (2017, p. 135) refere que o envelhecimento:

(...) implica uma responsabilização da própria pessoa e da sociedade em promover as ações necessárias para integrar uma perspectiva de mudança nos comportamentos e na capacidade de resposta do Governo ajustada ao ambiente e às necessidades.

No entanto, apesar dos passos dados, e da lógica de integração da pessoa idosa na sociedade, há ainda um campo que necessita de uma maior ação e que foca a integração da pessoa idosa com o desenvolvimento da sociedade digital de informação, para que as pessoas idosas não se sintam hoje, nem no futuro, infoexcluídas.

\section{Sociedade moderna, tecnologias de informação e risco de infoexclusão da pessoa idosa}

Na sociedade moderna, temos assistido a um rápido e recente crescimento das tecnologias de comunicação e informação e do acesso à internet. Os próprios serviços públicos em Portugal no âmbito da simplificação administrativa promoveram um conjunto de medidas que permitem o acesso aos dados do cidadão através de uma ligação da internet, permitindo solicitar documentos, comprovativos dos impostos, renovar documento de identificação, acessar aos dados de saúde, marcar consultas entre outras facilidades (Mateus, 2008).

A sociedade cada vez mais funciona numa rede tecnológica que gere e difunde o conhecimento e a comunicação através da realidade digital. Dessa forma, o ciberespaço tem ganho um grande destaque transpondo fronteiras, descentralizando e tornando o conhecimento global (Castells, \& Cardoso, 2005). 
Dessa forma, as relações humanas e a vida em sociedade tornam-se dependentes da adesão e utilização do espaço digital e, em decorrência disso, quem não se adaptar a esta nova realidade poderá ficar em situação de infoexclusão, perdendo sua integração e inclusão na sociedade (Castells, \& Cardoso, 2005).

As gerações mais recentes nasceram já com as novas tecnologias e desde muito cedo convivem com ferramentas e dispositivos eletrônicos (Bennett, Maton, \& Kervin, 2008). Na literatura internacional, a familiaridade com o mundo digital levou alguns autores (Prensky (2001) a apelidar esta nova geração de "Nativos Digitais", enquanto Tapscoot (1998) optara pelo termo "Geração da Internet". Numa visão oposta, os cidadãos que nasceram numa época em que as tecnologias de informação não existiam ou apenas estavam ao alcance de alguns, mas que tiveram de as adotar no seu dia a dia por motivos pessoais ou profissionais, foram chamados por Prensky (2001) de "Imigrantes Digitais".

Facilmente se pode entender que a forma como as tecnologias de informação são usadas depende do grau de utilização dado pelo utilizador. Nessa área, as pessoas idosas estão em desvantagem e integram um subgrupo especial entre os "imigrantes digitais", pelo facto de passarem décadas sem necessidade de usá-las, e, contrariamente aos nativos digitais, contatam uma nova realidade que é um acrescento a seu quotidiano (OberCom, 2010; Prensky, 2013). Por esse motivo, os mais idosos têm um conjunto de referenciais que não se integram facilmente num contexto digital. A este respeito, (Prensky, 2013, p. 24) afirma:

A tecnologia é uma extensão do nosso cérebro. É uma nova maneira de pensar. É a nova solução que nós, humanos, criamos para lidar com o nosso novo e difícil contexto de variabilidade.

Em Portugal, os dados relativos à utilização das tecnologias digitais são fornecidos pelo Instituto Nacional de Estatística (INE, 2015b). De acordo com esses dados, no ano de 2015 , cerca de $70 \%$ da população portuguesa utilizava computador e internet, e cerca de $23 \%$ recorria ao comércio digital. Contudo, os dados para a população idosa não são tão animadores. A figura seguinte apresenta os dados relativos à utilização de computador, internet e comércio digital. 
Figura 2 - Utilização de computador, internet e comércio digital (jovens vs idosos)

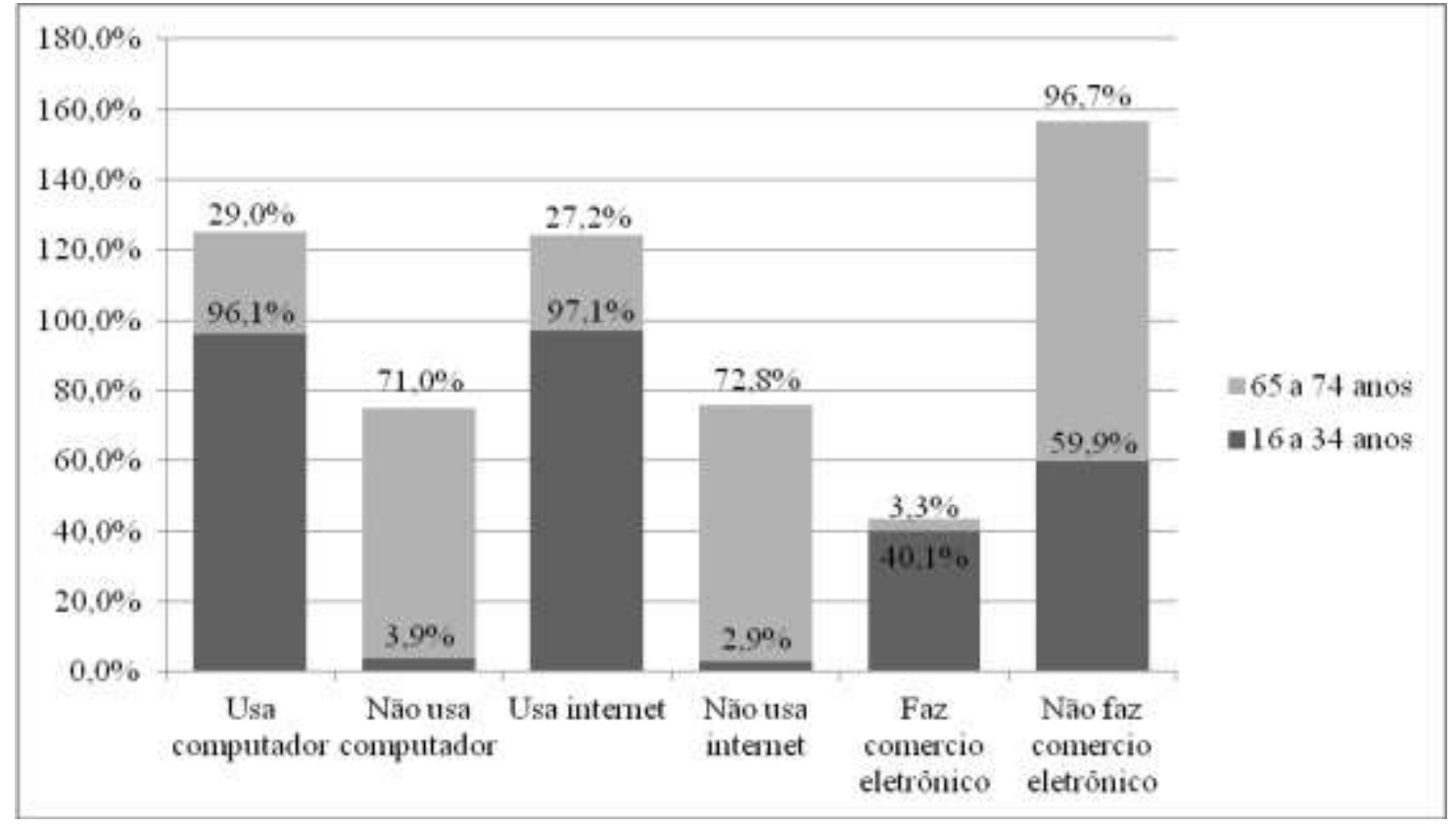

Fonte: INE (2015b)

Em geral, as pessoas mais idosas estão muito abaixo dos mais jovens em termos da utilização de computador (apenas 29\%), acesso à internet (apenas 27,2\%), e se realiza comércio eletrônico (apenas 3,3\%). Dessa forma, considerando estes dados, é notável a menor utilização das tecnologias digitais na população idosa relativamente aos mais novos, o que implica uma menor competência digital desse grupo etário.

A esse respeito, Carleto, e Santana $(2017$, p. 3) ratificam que:

Os estudos, que têm foco no uso de telefones celulares e outros equipamentos considerados como novas tecnologias digitais, demonstram que os idosos usam menos e têm mais dificuldades no manejo de novas tecnologias do que os jovens tanto no que se refere ao aprendizado destas quanto aos aspectos que envolvem o uso, tais como o medo de danificar o aparelho, o embaraço no uso destes equipamentos em público, a compreensão de como funcionam os equipamentos multifuncionais. 
Estes dados são preocupantes pelas razões já apresentadas, sendo evidente que as pessoas mais idosas, na maior parte das vezes, incluem-se num grupo de infoexcluídos, com todas as consequências que tal situação pode acarretar, existindo, portanto, a necessidade de promover a infointervenção no sentido de minimizar a infoexclusão.

Tendo em conta que a maioria dos idosos não teve ainda um contato com as tecnologias de informação, a União Europeia, em 2009, lançou um programa especialmente dedicado à aproximação dos mais idosos às tecnologias digitais "Ageing Well in the Information Society - i2010 Initiative", que tinha como objetivos compreender a utilidade dos sistemas digitais, e reduzir os efeitos da infoexclusão da pessoa idosa. Esta iniciativa assentava em três eixos de intervenção:

- Na comunidade (desenvolvimento de redes digitais para reduzir o isolamento social e promover o acesso aos serviços);

- No nível do trabalho (com a implementação de práticas inovadoras);

- No domicílio (com o objetivo de o idoso recorrer às tecnologias de informação digital no seu dia a dia, proporcionando-lhe melhor qualidade de vida e uma maior interatividade com a sociedade).

Os idosos com acesso aos meios digitais de comunicação deixarão de estar tão isolados da sociedade e poderão desenvolver estratégias para melhorar sua qualidade de vida e desenvolver habilidades/competências que os façam sentirem-se úteis à sociedade (Castells, \& Cardoso, 2005).

\section{A importância do desenvolvimento de uma estratégia de intervenção junto da pessoa idosa para adoção às redes digitais}

Tendo em conta os pressupostos do envelhecimento ativo, e em linha com a sociedade moderna, é importante desenvolver formas de familiarizar a pessoa idosa com os sistemas digitais, integrando essas atividades na sua vida diária. Para isso, é necessário conscientizar os idosos das vantagens que as tecnologias de informação podem ter na promoção de um modo de vida mais saudável e para sua integração ativa e participativa na sociedade (Mosquera, \& Strobaus, 2012). 
A abertura da pessoa idosa para a adoção das tecnologias digitais é um passo importante na luta contra a infoexclusão. Para isso, é necessária uma intervenção próxima dos Governos junto da pessoa idosa, desenvolvendo atividades, promovendo o acesso àqueles que terão menos recursos e conscientizando a todos sobre a importância dos sistemas digitais.

As tecnologias digitais predominam no mundo moderno e geram comunidades virtuais que permitem uma nova forma de interação com a realidade e com o mundo. Sobre este tema, Sebriam $(2009$, p. 37) teoriza que a relação entre as pessoas numa comunidade virtual:

(...) inaugura novas formas de sociabilidade, novas formas de interação na sociedade que partilham entre si um novo espaço de contornos muito especiais: o ciberespaço.

As limitações físicas decorrentes do envelhecimento incapacitam grande parte dos idosos que, por essa razão, têm dificuldade de marcha e de equilíbrio, o que impede que tenham uma vida social mais presente (Lacourt, \& Marini, 2006).

Por esse motivo, a integração da pessoa idosa em redes e grupos sociais permitirá, a partir do seu domicílio um contato com o mundo e uma participação ativa na sociedade, partilhando experiências e estratégias com outros idosos que se encontrem nas mesmas circunstâncias (Escobar-Bravo, Puga-González, \& MartínBaranera, 2012).

A esse respeito, Pasqualotti (2008, p. 24) complementa, referindo que "a interação social de troca de ideias e experiências de mundo (...) é construída pela participação na rede social”.

Além desta vantagem, de acordo com literatura, pode-se concluir que a adopção das tecnologias de informação e comunicação nesta era digital, pelos idosos, poderá também contribuir para uma maior interação com os mais novos, para conhecer as notícias, partilhar a sua opinião sobre o mundo e o país, e contribuir com sua experiência em grupos de debate, ao se conectar com todo o mundo através das redes sociais (Twitter, Facebook, Instagram...). 
Todas estas estratégias contribuem para a melhoria do estado mental e das componentes psicossocial e cognitiva (autoconceito, auto-estima e autorrealização), quebrando os estereótipos associados na perspectiva negativa do envelhecimento, e promovendo a inclusão e inserção do idoso na sociedade (Guy, Cleary, \& Henderson, 2010; Torres, \& Zagalo, 2007). A esse respeito, Esteves (2010, p. 6), complementa:

As limitações físicas do envelhecimento podem limitar muitas pessoas idosas que têm maior dificuldade em andar e ter equilíbrio o que impede uma mais presente vida social (Marini Lacourt, 2006). Por essa razão, a integração dos idosos em redes sociais e grupos permitirá que, em sua casa, um toque com o mundo, a participação ativa na sociedade, compartilhando experiências e estratégias com outros idosos que estão nas mesmas circunstâncias (Bravo-Escobar Puga-Gonzalez, \& Martín-Baranera, 2012).

A este respeito, Pasqualotti (2008, p. 24) refere que "a interação social para a troca de ideias e experiências no mundo (...) é construída através da participação na rede social", complementando ainda desta forma:

As Tecnologias de Informação e Comunicação podem apoiar as pessoas idosas a reduzir o seu isolamento e solidão, aumentando as possibilidades de se manterem em contato com familiares e amigos podendo, assim, alargar as suas redes sociais (Pasqualotti (2008, p. 24).

Os Governos devem promover a inclusão digital nas estratégias de integração da pessoa idosa na sociedade num momento que a informação digital e a tecnologia estão em grande crescimento, desde a utilização do computador, internet, correio eletrônico, compras e transferências bancárias on-line. Só com estas medidas será possível retardar alguns dos efeitos de infoexclusão no envelhecimento. 


\section{Considerações Finais}

Nas sociedades modernas, como é o caso de Portugal, o envelhecimento saudável e ativo determina a participação social da pessoa idosa em vários sectores da sociedade desde a saúde, educação, trabalho, cultura, justiça e desenvolvimento regional. Contudo, o desenvolvimento da era digital veio limitar o acesso das pessoas mais idosas ao mundo moderno pelo facto de não terem um contato frequente e familiarizado com as novas tecnologias.

Combater a infoexclusão é um desafio atual e futuro que se coloca e cada vez mais se colocará no desenvolvimento da sociedade que tem e deverá facilitar os processos por meio da informação digital e meios inovadores de interação com os serviços.

Também tem o dever de não discriminar nenhum cidadão e integrar e promover a pessoa idosa dentro dos novos desenvolvimentos, de forma a que estas não se sintam infoexcluídas e, por outro lado, a que usem esses meios para reforçar seu contato e envolvimento com adultos e jovens, promovendo, dessa forma, a intergeracionalidade.

Pelas razões apresentadas, é importante que o Governo desenvolva medidas e estratégias para promover uma cultura digital junto das pessoas mais idosas. Este será um dos objetivos de Portugal para os próximos anos que, no âmbito da modernização administrativa, não deixará de fora as pessoas mais idosas, e promoverá uma simplificação administrativa adequada a esta população através dos meios digitais.

\section{Referências}

Albert, M., Jones, K., Savage, C., Berkman, L., Seeman, T., Blazer, D., \& Rowe, J. (1995). Predictors of cognitive change in older persons: MacArthur studies of successful aging. Psycholy Aging, 10(4), 578-589. Recuperado em 01 fevereiro, 2017, de: https://www.ncbi.nlm.nih.gov/pubmed/8749585.

Bengtson, V., Gans, D. Putney, N., \& Silverstein, M. (2009). Theories about age and aging. In Bengtson, V., Gans, D., Putney, N. \& Silverstein, M. (Eds.). Handbook of theories of aging, 02-24. Nova York, USA: Springer.

Bennett, S., Maton, K., \& Kervin, L. (2008). The 'digital natives' debate: A critical review of the evidence. British Journal of Education Technology, 39(5), 775-786. Recuperado em 01 fevereiro, 2017, de: doi: 10.1111/j.1467-8535.2007.00793.x. 
Brito, A. (1993). Psicologia, idosos e exercício. In: Marques, A., Gaya, A., \& Constantino, C. (Eds.). Physical activity and health in the elderly. Proceedings of the first conference of European Group for Research into Elderly and Physical Activity, 102-116. Porto, Portugal: Universidade do Porto.

Bruno, F. (2006). O biopoder nos meios de comunicação: o anúncio dos corpos virtuais. Revista da Escola Superior de Propaganda e Marketing, Comunicação, mídia e consumo, 3(6), 63-79. Recuperado em 01 fevereiro, 2017, de: http://revistacmc.espm.br/index.php/revistacmc/article/view/59.

Carleto, D. G., \& Santana, C. da S. (2017). Relações intergeracionais mediadas pelas tecnologias digitais. São Paulo (SP): PUC-SP: Revista Kairós Gerontologia, 20(1), 73-91. Recuperado de: doi: 10.23925/2176-901X.2017v20i1p73-91.

Carvalho, M. (2012). Envelhecimento e cuidados domiciliários em instituições de solidariedade social. Lisboa, Portugal: Coisas de Ler.

Carvalho, M. (2013). Serviço Social no Envelhecimento. Lisboa, Portugal: LIDEL.

Castells, M., \& Cardoso, G. (2005). A Sociedade em Rede - do conhecimento à Acção Política. Lisboa, Portugal: Imprensa Nacional da Casa da Moeda.

CENSUS. (2011). Census da população portuguesa. Lisboa, Portugal: Instituto Nacional de Estatística.

Cumming, E., \& Henry, W. (1961). Growing Old. New York, USA: Basic.

Duarte, V., Santana, M., Soares, M., \& Thofern, M. (2005). A perspectiva do envelhecer para o ser idoso e sua família. Família Saúde e Desenvolvimento, 7(1), 4250. Recuperado em 01 fevereiro, 2017, de: http://revistas.ufpr.br/refased/article/viewFile/8052/5674.

Ebersole, P. (2005). Gerontological Nursing \& Healthy Aging. Estados Unidos: Elsevier Mosby.

Escobar-Bravo, M, Puga-González, D., \& Martín-Baranera, M. (2012). Protective effects of social networks on disability among older adults in Spain. Archives of Gerontology and Geriatrics, 54(1), 109-116. Recuperado em 01 fevereiro, 2017, de: https://doi.org/10.1016/j.archger.2011.01.008.

Esteves, S. (2010). Envelhecer bem com as tecnologias de informação e comunicação: uma abordagem aos desafios do envelhecimento e às oportunidades das TIC. Cidade Solidária, 23, 64-69. Recuperado em 01 fevereiro, 2017, de: http://suti.ics.lisboa.ucp.pt/pacweb/SearchResultDetail.aspx?mfn=22209\&DDB=\#.W ZoVxT6GPik.

Fernandes, A. (1999). Velhice e Sociedade: alterações nos calendários demográficos e políticas sociais. In: V Cursos internacionais de Verão de Cascais - A Família, 21-59.

Fonseca, A. (2005). O envelhecimento bem-sucedido. In: Paúl, C., \& Fonseca, A. (Eds.). Envelhecer em Portugal, Psicologia, Saúde e Prestação de Cuidados, 281309). Lisboa, Portugal: Climepsi Editores.

Freire, S. (2000). Envelhecimento bem-sucedido e bem-estar psicológico. In: Neri, A., \& Freire, S. (Eds.). E por falar em boa velhice, 21-31). Campinas, SP: Papirus.

Guy, A., Cleary, K., \& Henderson, C. (2010). Use of the Nintendo WiiTM with older adults in the skilled nursing facility setting. GeriNotes, Cherry Hill, 17(3), 05-08. 
INE. (2015a). Estatísticas da população residente, Portugal e NUTS III, 2015. Lisboa, Portugal: Instituto Nacional de Estatística.

INE. (2015b). Sociedade da Informação e do Conhecimento - Inquérito à Utilização de Tecnologias da Informação e da Comunicação nas Famílias. Lisboa, Portugal: Instituto Nacional de Estatística.

Lacourt, M., \& Marini, L. (2006). Decréscimo da função muscular decorrente do envelhecimento e a influência na qualidade de vida do idoso: uma revisão de literatura. Revista Brasileira de Ciências do Envelhecimento Humano, 3(1), 114-121. Recuperado em 01 fevereiro, 2017, de: http://seer.upf.br/index.php/rbceh/article/view/51.

Lima, M. (2010). Envelhecimento(s), Estado da Arte. Coimbra, Portugal: Imprensa da Universidade de Coimbra.

Mateus, J. (2008). O Governo Electrónico, a sua aposta em Portugal e a importância das Tecnologias de Comunicação para a sua estratégia. Polytechnical Studies Review, 6(9), 01-05.

Mosquera, J., \& Strobaus, C. (2012). O envelhecimento saudável: educação, saúde e psicologia positiva. In: Ferreira, A., Stobaus, C., Goulart, D., \& Mosquera, J. (Eds.). Educação e Envelhecimento, 14-22. Porto Alegre, RS: ediPUCRS.

Nazareth, J. (1988). Portugal, os próximos 20 anos: unidade e diversidade da demografia portuguesa no final do século XX. Lisboa, Portugal: Fundação Calouste Gulbenkian.

Novais, M. (2008). Paradoxos Contemporâneos. Rio de Janeiro, RJ: E-papers.

OberCom - Observatório da Comunicação (2010). Nativos digitais portugueses Idade, experiência e esferas de utilização das TIC. Lisboa, Portugal: Obercom.

Nunes, A., \& Nunes, M. (2016). A saúde em Portugal: um olhar sobre o distrito de Castelo Branco. Portugal: RVJ Editores.

Nunes, A. (2017). Demografia, envelhecimento e saúde uma análise ao interior de Portugal. Revista Kairós Gerontologia, 20(1), 133-154. São Paulo, SP: PUC-SP. Recuperado em 17 maio, 2017, de: https://revistas.pucsp.br/index.php/kairos/article/view/32413. Doi: 10.23925/2176-901X.2017v20i1p133-154.

OMS - Organização Mundial de Saúde (2002). Organização Mundial de Saúde. Active ageing: a policy framework. A contribution of the WHO to the Second United Nations World Assembly on Ageing. Madrid, ES: OMS Publications.

ONU (2002). Organização das Nações Unidas. International Plan of Action on Ageing 2002. In: 2. ${ }^{a}$ Assembleia Mundial sobre o Envelhecimento. Madrid, ES: ONU.

Pasqualotti, A. (2008). Comunicação, tecnologia e envelhecimento: significação da interação na era da informação. Tese de doutorado em Informática na Educação. Porto Alegre, RS: Universidade Federal do Rio Grande do Sul.

Prensky, M. (2001). Digital natives, digital immigrants. On the Horizon, 9(5), 01-06. Recuperado em 01 fevereiro, 2017, de: https://www.marcprensky.com/writing/Prensky\%20\%20Digital\%20Natives, \%20 Digital\%20Immigrants\%20-\%20Part1.pdf.

Prensky, M. (2013). Our brains extended. Technology-Rich Learning, 70(6), 22-27. Recuperado em 01 fevereiro, 2017, de: http://www.ascd.org/publications/educationalleadership/mar13/vol70/num06/Our-Brains-Extended.aspx. 
Quaresma, M. (2008). Questões do envelhecimento nas sociedades contemporâneas. Revista Kairós Gerontologia, 11(2), 21-47. São Paulo (SP): PUC-SP. Recuperado em 06 março, 2017, de: https://revistas.pucsp.br/index.php/kairos/article/view/2391/1484.

Quaresma, M. de L., \& Ribeirinho, C. (2016). Envelhecimento - Desafios do Séc. XXI. Revista Kairós Gerontologia, 19(3), 29-49. São Paulo (SP): PUC-SP.

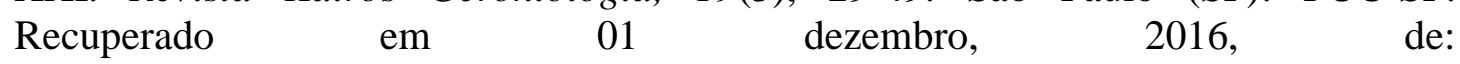
https://revistas.pucsp.br/index.php/kairos/article/view/30900/21382.

Sebriam, D. (2009). Utilização das tecnologias de informação e comunicação no ensino da educação física. Dissertação de mestrado em Engenharia de Mídias para a Educação. Lisboa, Portugal: Faculdade de Motricidade Humana da Universidade Técnica de Lisboa.

Sequeira, C. (2007). Cuidar de Idosos Dependentes. Coimbra, Portugal: Quarteto Editora.

Silva, M. E. V. da. (2006), Se fosse tudo bem, a velhice era boa de enfrentar: Racionalidades leigas sobre envelhecimento e velhice - um estudo no Norte de Portugal. Tese de doutorado. Porto, Portugal: Universidade Aberta.

Sousa, L., \& Figueiredo, D. (2003). (In)dependência na população idosa: um estudo exploratório na população portuguesa. Psychologica, 3, 109-120.

Tapscoot, D. (1998). Growing Up Digital. The Rise of the Net Generation. Nova York, USA: McGraw Hill.

Torres, A., \& Zagalo, N. (2007). Videojogos: um novo meio de entretenimento de idosos? In: Congresso da Associação Portuguesa de Ciências da Comunicação, 21672175). Braga, Portugal: Centro de Estudos de Comunicação e Sociedade, Universidade do Minho.

Tredinnick, L. (2008). Digital Information Culture. Estados Unidos: Elsevier Chandos Publishing.

Walsh, F. (1999). Families in later life: challenges and opportunities. In: Carter, B., \& McGoldrick, M. (Eds.). The expanded family life cycle: individual, family, and social perspectives, 307-326. Boston, USA: Allyn \& Bacon.

Recebido em 12/06/2017

Aceito em 30/06/2017

Nunes, A. M. (2017). Modernização, envelhecimento e infoexclusão em Portugal. Revista Kairós - Gerontologia, 20(2), pp. 79-99. ISSNe 2176-901X. São Paulo (SP), Brasil: FACHS/NEPE/PEPGG/PUC-SP 
Alexandre Morais Nunes - PHD em Ciências Sociais, Especialidade de Administração da Saúde, Instituto Superior de Ciências Sociais e Políticas (ISCSP), Universidade de Lisboa. Mestre em Administração da Saúde, ISCSP, ULISBOA, Portugal. Professor Auxiliar Convidado no Instituto Superior de Ciências Sociais e Políticas (ISCSP), Universidade de Lisboa. Assessor Técnico/Technical advisor do Ministro da Saúde. Área da Administração de Serviços de Saúde. Ministério da Saúde de Portugal. Lisboa, Portugal.

E-mail: anunes@iscsp.ulisboa.pt; alexandre.nunes@ms.gov.pt 